\title{
Determination of a Short Simulation Sequence for the Multi-Criteria Optimization of Buildings: A Case Study
}

\author{
Hasan Sayegh ${ }^{12}$, Antoine Leconte ${ }^{2}$, Gilles Fraisse ${ }^{1}$, Etienne Wurtz ${ }^{2}$, Simon Rouchier ${ }^{1}$ \\ ${ }^{1}$ Laboratoire Optimisation de la Conception et Ingénierie de l'Environnement, Chambéry, France \\ ${ }^{2}$ Laboratoire d'Innovation pour les Technologies des Energies nouvelles et les Nanomatériaux \\ (LITEN), CEA Tech, Chambéry, France
}

\begin{abstract}
A new holistic approach of system optimization based on the reduction of simulation period rather than the model itself is a current subject of interest in the domain. This paper starts by comparing precision and efficiency of the reduction approaches used in the literature, the clustering approach and the iterative method are found to be the most practical. Further analysis is performed on the iterative approach. It proved its efficiency in estimating annual performance while monthly performance not accurately estimated. Moreover, a parametric analysis is done on this approach to validate its stability and showed that it is sensitive to the size and thermal inertia of the system and therefore not capable to be generalized but requires further enhancement.
\end{abstract}

\section{Introduction}

Globally, the building sector is one of the largest consumers of energy and accounts for about $40 \%$ of the total energy consumption (Pérez-Lombard, Ortiz and Pout, 2008). Energy consumption and demand of services increase with the growth in economy and population rising the building energy demand to the levels of transport and industry.

However, the building sector offers significant potential for improved energy efficiency with highperformance envelops and energy-efficient systems. From this point, the interest in building design optimization has become the trend of the domain. Optimization algorithms have been used because of the need for energy system designs that minimize costs and environmental impact. Studies have been done on many parameters that play a role on the building performance and may improve its impacts. Yet, performing holistic approaches on buildings, which takes into consideration both the envelope and the systems, leads to the complexity of models under study, especially when analyzing heat networks in the case of multiple buildings i.e. districts or blocks, leading therefore to unfeasible computational time expenses. Usually, this issue is solved by reducing the complex models into simplified ones, as for example neglecting some decision parameters, using analogical RC models or proposing meta-models such as artificial neural networks or kriging models. A new holistic approach that might solve those doubts is a current case of interest. It is based on the reduction of period of simulation rather than the model itself. The approach evaluates annual performance starting from a short sequence of typical selected days as representatives of other similar ones as shown in Figure1.

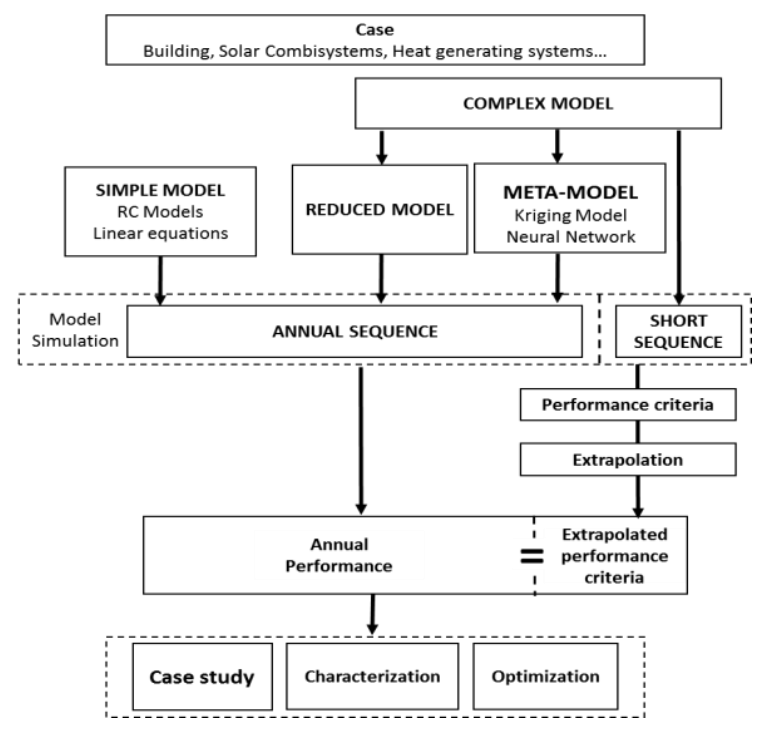

Figure 1: The relation between the complexity of the case study and the type of time sequence used for simulations.

However, several questions rise concerning the credibility of this method and its capability to estimate annual performances from a reduced period of time. How much computational time can be economized? Is it applicable to all case studies or 
limited to specific ones by itself? Is the short sequence stable enough to assess, starting from certain criteria, other ones not taken into consideration when constructing the short sequence? What about the criteria that are related to the dynamism of a simulation, such as thermal inertia? To try to reply to all those questions and other, this paper starts by presenting the main approaches find in the literature regarding their method of functionality and the comments put by the researchers. One of those approaches is then selected for further assessments by analyzing its performances and performing parametric analysis to assess its stability in system model studies. Finally, the conclusion wraps up the lessons learnt and implements several perspectives for future research work

\section{Sequence Reduction Methods}

The literature contains various methods to select a representative set of historical periods. The process starts by the original annual data and ends in a short sequence that will be later used in system study, system characterization or system optimization. The selection criteria depend on the evaluated attributes. Usually weather data and load curves are taken into consideration. Other criteria could be used such as maximum power and internal temperature. These selection criteria can have several profiles such as temporal curves, cumulative curves, scatter of points or unitary recordings.

The methods can be grouped in three large categories: Heuristic Methods, Iterative Methods and Grouping Algorithms.

\section{$\underline{\text { Heuristic Methods }}$}

When finding an optimal solution is impossible or impractical, heuristic methods can be used to speed up the process of finding a satisfactory solution. The heuristic method is a problem solving that employs a practical method, not guaranteed to be optimal but sufficient for reaching an immediate goal. The idea behind most of simple heuristic methods is to select a number of periods with different load and/or meteorological conditions in order to capture a variety of different events, Figure 2. Belderbos (2015), Fripp (2012) and Hart (2011) reduced the data size of building performance by selecting specific days that contain hours with extreme meteorological and load events to characterize typical system behavior.

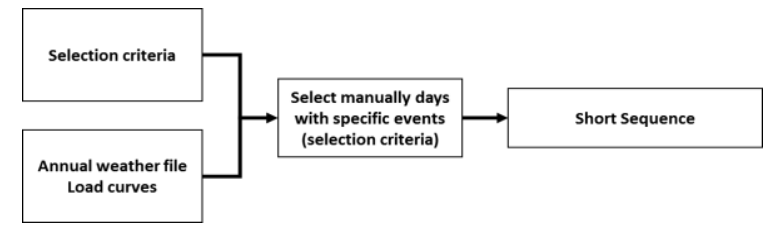

Figure 2 Heuristic method in typical day selection.

\section{Iterative Methods}

The iterative method is an approach that searches for the best solution after repeating the same action several times and comparing the quality of results in each iteration, Figure 3. There are many examples in the literature that use this method for day selection, either directly by implementing iterations or indirectly through performing graphical methods as the work done by Ortiga, Bruno and Coronas (2011) or through performing Mixed Integer Linear Programing (MILP) based iterations.

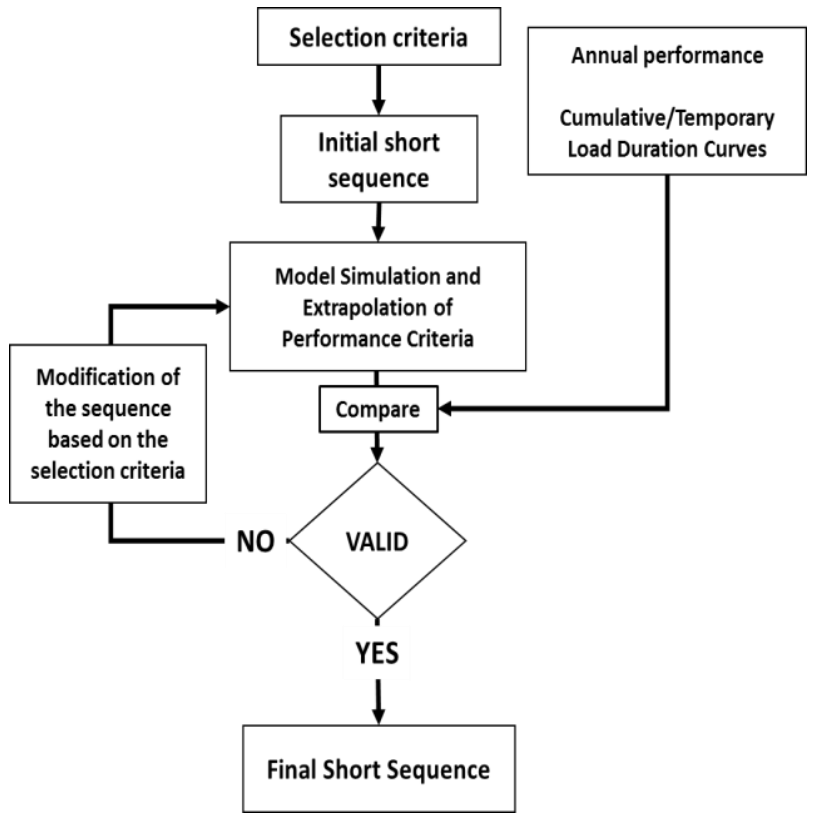

Figure 3: Iterative method in typical day selection.

The French Commission of Alternative Energies and Atomic Energy (CEA) has developed an iterative method that reduces a whole year into twelve days and was used for testing a solar combisystem. The method was called Short Cycle System Performance Test (SCSPT), which selects the short sequence based on the energy demand and energy stored in the system. Results were very promising and the sequence was able to reproduce the annual performance with a good degree of accuracy applicable for different models (Albaric, Nowag, and Papillon, 2008).

\section{Grouping Algorithm}

More advanced approaches to select a representative set of historical periods employ grouping algorithms. Days with similar attributes are grouped into clusters followed by day selection of each group, Figure 4 . While clustering algorithms were the most preferred in studies (Fazlollahi and Maréchal, 2014; Menegon, Soppelsa, and Fedrizzi, 2017; Ribault, 2017), some studies employed discriminant analysis, to achieve grouping (Balachandra., 1999). 


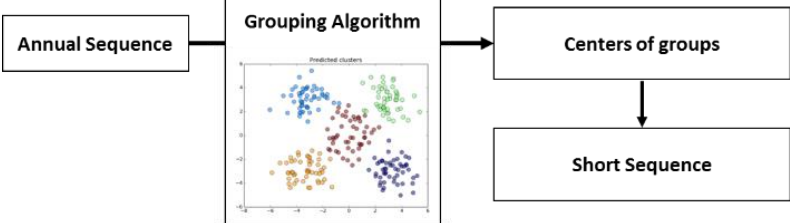

Figure 4: Grouping method in typical day selection.

\section{Extrapolation of Results}

The process of time reduction is also directly related to the way of extrapolation of the results found by the selected days. The adequacy of the extrapolated results and their proximity to the real values are the indicators for the success or failure of the method. Extrapolation is usually performed by two main methods: multiplying the obtained results by a proportion or multiplying the obtained results by the weight of the group represented by a day. While the former is usually used in heuristic and iterative approaches, it is simpler yet less accurate logically than the latter. Multiplying by a proportion is the multiplication of the results of a short simulation sequence by a single scalar depending on the number of days in the short sequence, so it gives the same effect for all the days of the sequence regardless of their real representation. On the other hand, extrapolation by actual group weight multiplies each value by a unique scalar that depends only on the weight of the group it came from. This way of extrapolation is inevitable in clustering algorithms where the scalar depends on the size of each cluster

\section{Comparison of the Methods}

In the literature, it is noticeable the predilection by the researchers into using clustering algorithms rather than other methods, with a special interest in the K-means clustering method. This interest is due to its good performance in achieving the objective. The computational time expense was least in heuristics and clustering algorithms. Analysis done by researchers showed the importance of considering peak periods for improving the accuracy of the study where adding them as isolated clusters will represent the days with extreme demands and therefore enhance the obtained results. Heuristic method is the simplest one of reduction algorithms where no difficult coding with complicated math equations is required through the procedure. However, when it comes to the precision of the obtained results after simulating the short sequence and comparing its compatibility with the annual sequence, the performance of the heuristic approach is the worst (Kotzur L. and Markewitz P.(2018)). Expecting precise results from a heuristic method requires high experience by the operator to get an efficient sequence and not wasting time by trial and error. This is not found in clustering and iterative cases where it is left for the computer to do all the trials and give finally the selected sequence, with a higher precision, and a shorter period.

On the other hand, the diversity of the case studies found in the literature does not make us able to favor a method over another. The efficiency of the method is directly related to the system studied or optimized. However, it was shown that the same method could be used to study several case studies, which is a major benefit in the sake of defining a new approach to be used in optimization studies. Where in such studies, continuous modification of model parameters takes place throughout the optimization procedure. Therefore, the use of an approach that shows stability despite the modifications is necessary.

For that reason, further study was done on the iterative approach SCSPT to assess its stability and parametric analysis was performed to define its efficiency.

\section{Case Study}

The solar combisystem model tested is presented in Figure 5 and is explained by Cheze D. et al (2014). It supplies energy for a two story residential building constructed in Le Bourget du Lac, France.

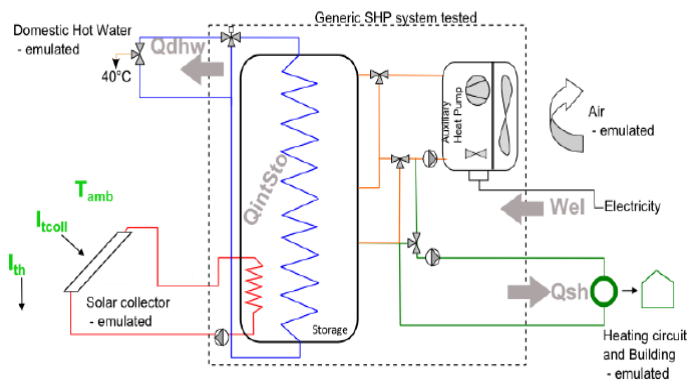

Figure 5: Solar combisystem model.

\section{Assessment of SCSPT}

In order to build the short sequence, SCSPT uses three monthly climate criteria in addition to several monthly performance criteria as attributes for the calculation of the selection criteria. The three selection criteria are:

- A "Target Ambient Temperature" $T$ ' ${ }_{a m b}$ calculated from the monthly ambient temperature $\left(\mathrm{T}_{\mathrm{amb}}\right)$, the monthly space heating energy consumption ( $Q_{\text {heating}}$ ) for the heating season and the monthly internal temperature $\left(T_{\text {int }}\right)$ for the cooling season.

- A “Target Total Irradiation Sum" $G$ ' coll on the collector's surface calculated from the monthly total solar irradiation $\operatorname{sum}\left(G_{\text {coll }}\right)$, the monthly energy stored in the $\operatorname{tank}\left(Q_{\text {sto }}\right)$, the electrical energy needed $\left(Q_{a u x}\right)$ for the 
heating season and the energy need for cooling $\left(Q_{\text {cooling }}\right)$ in the cooling season.

- A "Target Total Horizontal Irradiation Sum" $G$ 'hor calculated from the monthly total horizontal irradiation sum $\left(G_{h o r}\right)$ and the monthly internal temperature $\left(T_{i n t}\right)$.

$\bullet$

The algorithm then searches for real annual days that would have the closest criteria to those three criteria by calculating a global error $\Delta \mathrm{E}$ and limiting it to a threshold $\gamma$.

These steps are repeated continuously for each month until constructing the 12 days simulation sequence.

In order to assess the efficiency of the SCSPT algorithm, we analyzed three criteria used in the calculation of the selection criteria:

- $\quad$ Electrical energy needed $Q_{a u x}$

- Energy stored in the Tank $Q_{s t o}$

- Energy for space heating $Q_{\text {heating }}$

Those three criteria would give an idea about the energy aspect of the system.

We also analyzed a forth criterion that was not used in the selection process

\section{- Collector's temperature $\mathrm{T}_{\text {coll }}$}

This criterion would give an idea about the durability of the system through the distribution of the collector's temperature over the sequence, which is also a performance criterion under interest in model studies.

Analyzing the results of the reference model with a collector surface of $9.3 \mathrm{~m}^{2}$ and a storage tank volume of $0.8 \mathrm{~m}^{3}$, it is clear from Figures 6-8 that the SCSPT algorithm is able to estimate very closely the annual sum of the energetic criteria with errors inferior to $10 \%$. However, reproducing similar values to the monthly ones is not very efficient where there is always noticeable gaps in the plots and the coefficient of determination $\left(\mathrm{R}^{2}\right)$ ranged between 0.59 and 0.68 . Moreover, despite the temperature of the collector was not taken into consideration while the development of the SCSPT method, Figure 9 shows that it is still able to predict the annual recordings of the collector's temperature with a relatively good precision and an $\mathrm{R}^{2}$ of 0.827 . However, the discrepancies in the estimation of picks might be reasoned by the adopted method of extrapolation. In SCSPT, extrapolation is done by a scalar of $365 / 12$, thus giving equal weights to all representative days which is not the case in reality and might lead to over prediction.

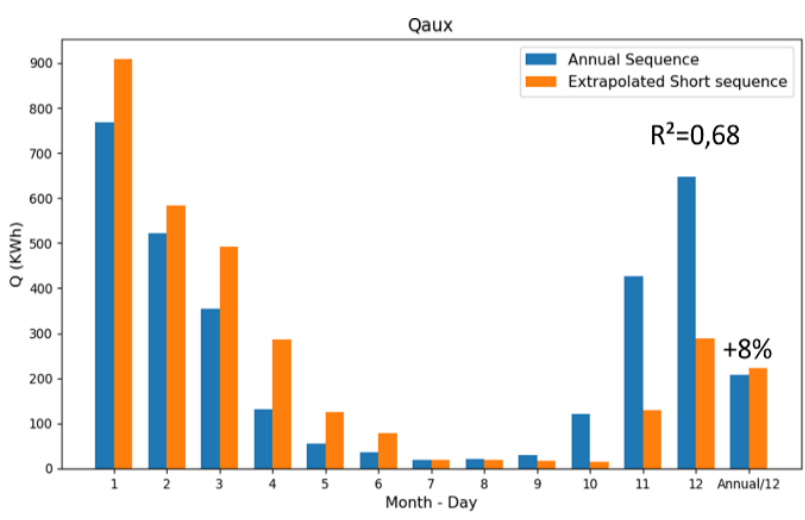

Figure 6: Estimation of the electrical energy.

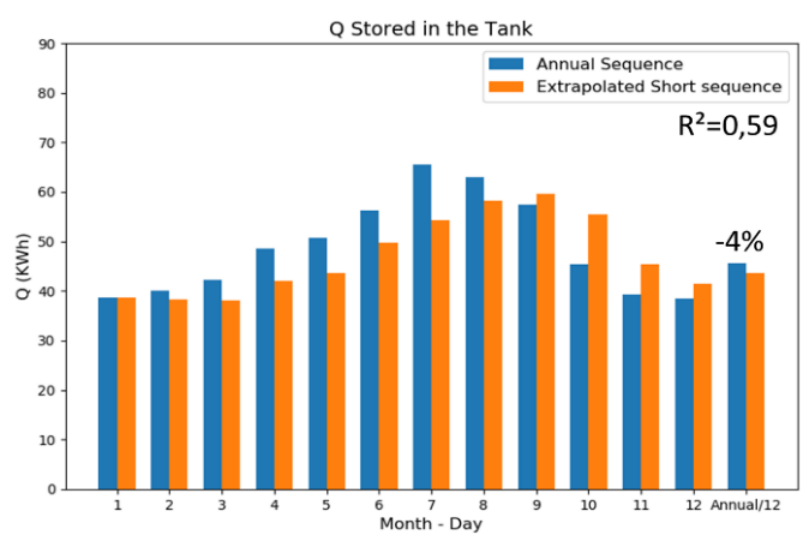

Figure7: Estimation of the energy stored in Tank.

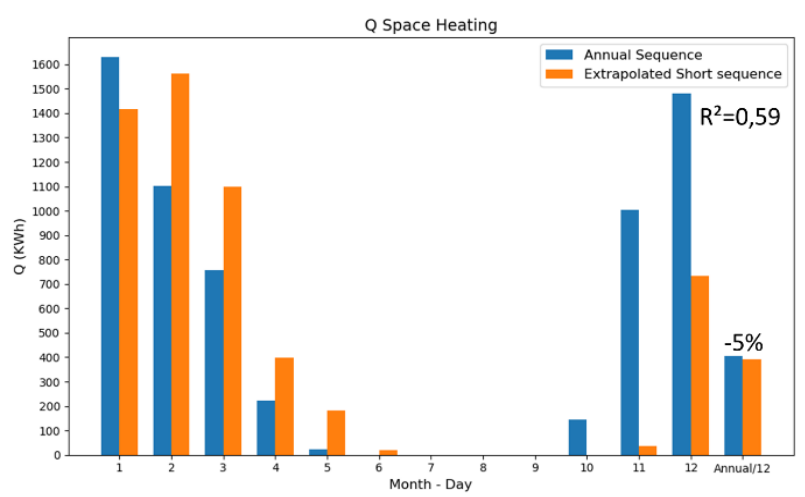

Figure 8: Estimation of the space heating energy.

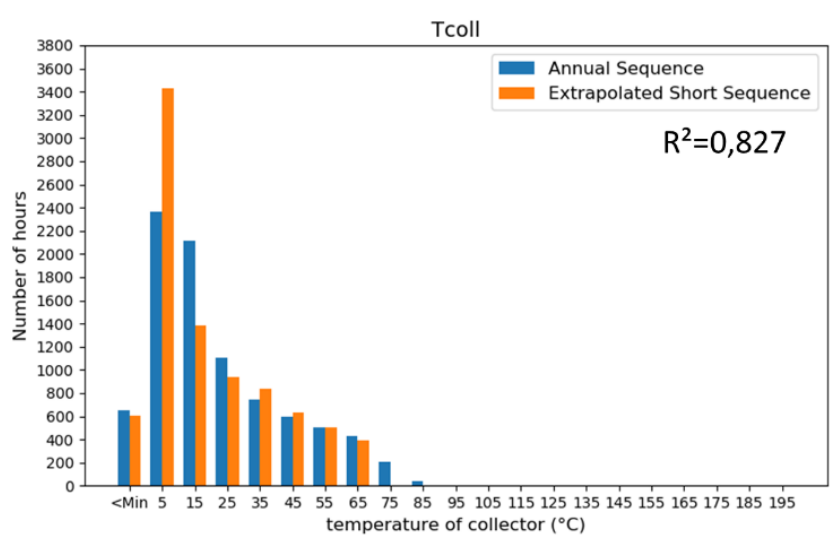

Figure 9: Estimation of the recorded temperatures of the collector. 


\section{Generalization Assessment}

In order to check if the approach still works upon changing of the case study, a generalization assessment is required. Therefore, a parametric analysis is done using Latin Hypercube Sampling (LHS) as a statistical method of sampling. The surface of the solar collector and the volume of the storage tank were modified. The aim is to assess the stability of the approach and verify that the generated sequence is still capable in estimating the annual performances. The study consists in generating three new short simulation sequences of 12 days for three new solar combisystem models (modifying the surface of collector $S$ and the volume of the storage tank $V$ ). Then, for each short sequence, the parametric modification is performed. Therefore, four cases in total to generate the short sequence were tested.

- Case 1(Reference): $V=0.8 \mathbf{~ m}^{3} \mathrm{~S}=\mathbf{9 . 3} \mathrm{m}^{2}$

- Case 2: $V=\mathbf{1 . 3} \mathbf{~ m}^{\mathbf{3}} \quad \mathrm{S}=\mathbf{2 0 \mathrm { m } ^ { 2 }}$

- Case 3: $V=\mathbf{0 . 6} \mathbf{~ m}^{\mathbf{3}} \quad \mathrm{S}=\mathbf{5} \mathrm{m}^{\mathbf{2}}$

- Case 4: $V=\mathbf{0 . 3} \mathbf{~ m}^{3} \quad \mathbf{S}=\mathbf{1 7 . 5 m ^ { 2 }}$

Table 1 shows the different samples that were tested for each case with "Sample 7" being the sample of the original model.

Table 1: Different samples studied in the parametric analysis.

\begin{tabular}{|c|c|c|}
\hline Sample & $\begin{array}{c}\text { Collector } \\
\text { Surface } \\
\left(\mathbf{m}^{2}\right)\end{array}$ & $\begin{array}{c}\text { Storage Volume } \\
\left(\mathbf{m}^{3}\right)\end{array}$ \\
\hline 1 & 7.5 & 0.3 \\
\hline 2 & 17.5 & 0.3 \\
\hline 3 & 5 & 0.5 \\
\hline 4 & 12.5 & 0.5 \\
\hline 5 & 17.5 & 0.7 \\
\hline 6 & 20 & 0.7 \\
\hline 7 & 9.3 & 0.8 \\
\hline 8 & 7.5 & 0.9 \\
\hline 9 & 12.5 & 0.9 \\
\hline 10 & 15 & 0.9 \\
\hline 11 & 5 & 1.1 \\
\hline 12 & 20 & 1.1 \\
\hline 13 & 7.5 & 1.3 \\
\hline 14 & 17.5 & 1.3 \\
\hline 15 & 10 & 1.5 \\
\hline 16 & 12.5 & 1.5 \\
\hline
\end{tabular}

Figures 10-14 show respectively the results obtained in each case for total auxiliary energy estimation
Qaux, Energy stored in tank estimation $Q_{s t o}$, Space heating estimation $\mathrm{Q}_{\text {heat }}$ recorded hours of collector temperature above $45^{\circ} \mathrm{C}$ estimation and the recorded hours of collector's overheating estimation (collector temperature $>100^{\circ} \mathrm{C}$ ). Each tested short sequence is assigned a shape on the plots i.e. Short Sequence 1: "X", Short Sequence 2: “+”, Short Sequence 3: "•" and Short Sequence 4: “ $\boldsymbol{\Delta}$ ". The different samples from Table 1 are listed in an increasing order of the volume of the tank. They are assigned colors in order to have a visual idea about the effect of the modifications on the accuracy of the obtained results. The positions of the results obtained in the case of original models on which the short sequence was generated for each of the four cases are denoted "SS" as in Short Sequence and indicated on the figures.

The plots show that the accuracy varies for each case and the method does not work for all system models. More precisely for systems with large equipment which was witnessed in Case 2. For the other cases, the estimation of the selection criteria (Qaux, Q stored in tank and Q space heating) were very good estimated with errors inferior to $10 \%$. It was noticeable from Figures 10, 11, 12 and 13 that better energy demand estimation is achieved in the case of smaller storage volumes. This might be due to thermal inertia of the tank, the smaller the tank is the lesser the thermal inertia is. Moreover, despite the temperature of the collector was not taken into consideration while the development of the SCSPT code, Figure 13 shows again that it is still able to predict the recordings of the annual performance despite the precision is not very high with errors exceeding slightly the $10 \%$ limit. The shift from the bisector in the figures could be improved by changing the method of extrapolation to a better one. However, Figure 14 shows that SCSPT does not allow the prediction of the durations of the collectors' overheating in all cases and for all samples.



Figure 10: Parametric analysis results of electrical energy need estimation Qaux. 


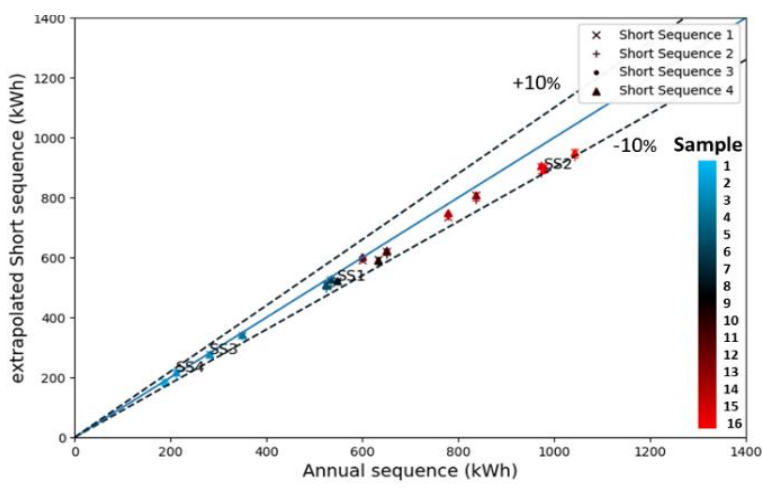

Figure 11: Parametric analysis results of energy stored in tank estimation.



Figure 12: Parametric analysis results of space heating energy estimation.

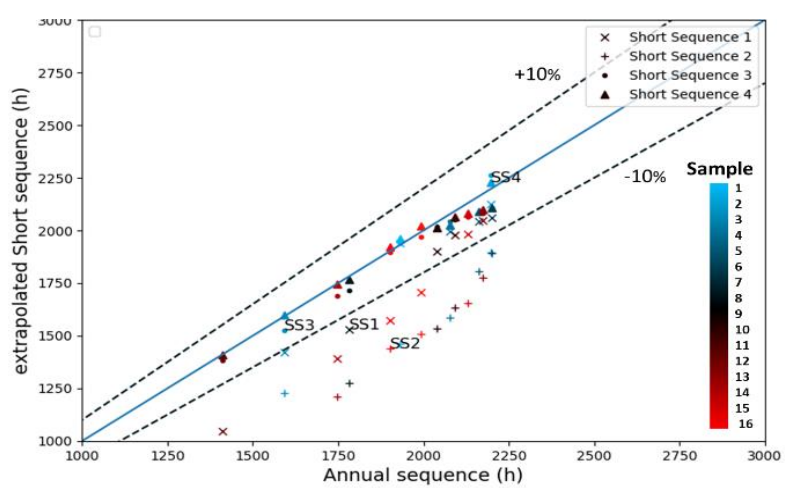

Figure 13: Parametric analysis results of estimating temperature of collector above $45^{\circ} \mathrm{C}$.

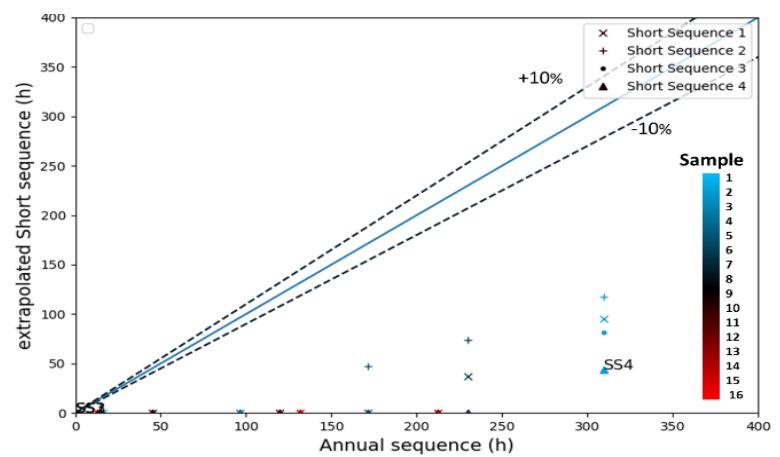

Figure 14: Parametric analysis results for collector overheating instants estimation.
Assessing the solar collector's overheating is considered interesting in the case of system design because of the idea it gives about the durability of the system. The overheating phenomenon affects severely the life of the solar panel due to the high pressure and temperature condition that can damage the system over time. Therefore, having the ability of estimating it will give the ability of designing models that avoid the phenomenon of stagnation. However, as shown in figure 14, the SCSPT method is unable to predict it. For example, estimation of the recorded hours of overheating of Sample 12 for Case1 is shown in Figure 15.



Figure 15: Distribution of the collectors' temperature in Sample 12.

The phenomenon of overheating took place mostly in systems with equipment that are oversized, when the collector's area is very big compared to the volume of the tank and the withdrawal of hot water. The phenomenon of thermal inertia of the large tank might be standing behind this failure in predicting the instants of high temperature. Where it is difficult for an approach that is based on several discrete days to estimate a physical phenomenon that takes a period of several consecutive days to attain stability. Therefore, an improvement in the selection criteria adding days with more sun exposure might cover the effect of thermal inertia and would give a more realistic case for simulation.

\section{Computational time expenses}

The main purpose in using short simulation sequences in building simulation studies is the reduction of the computational time expenses. It was noticed when recording the time of simulation the major impact of this approach. An annual simulation with Trnsys of the model showed in Figure 5 took about 19 minutes. On the other hand, a simulation on the 12 days sequence took 41 seconds. The time of reduction was reduced by about 28 times. Similarly, a complete parametric analysis of 16 cases (Table 1) took about four and a half hours for an annual simulation while 15 minutes for an analysis based on the short simulation sequence. This great reduction 
in time of simulation was accompanied by a good annual sum prediction of the studied criteria but less accurate monthly estimations as shown in the previous figures. So, using such concept in optimization studies, but with enhanced approaches capable of estimating both annual and monthly values and applicable on different case models will be a breakthrough in this domain for its reduction of the computational time expenses.

\section{Discussion}

Thanks to the statistical study and the parametric analysis performed, we were able to have a general yet rich overview on one of the considered to be "developed" approaches used in the concept of model simulation by short cycle sequences. And therefore have the ability to clarify some of the many questions that we presented them in the beginning of this paper. We can say that the method is indeed capable to economize a lot of time of simulation in addition to estimating the annual sum or global value of several performance criteria considered important for any system model assessment. However, it is found to be unreliable when it comes to partial or monthly estimations and in estimating criteria which were not taken into consideration when developing the short sequence. That in addition to not being able to overpass the effect of thermal inertia. So, the method might be used, as it is, if the aim is to study very complex and specific models without oversized equipment, which usually have high computational time expenses, but with relatively low thermal inertia, so it won't impact severely the accuracy of the obtained results. Moreover, while the generalization of the method was not found to be an option, modifying the way of selection and extrapolation by freeing the way of day selection from the empirical equations it is based on, might improve the quality of the sequence making it more stable upon parametric modifications.

\section{Conclusions and Future Work}

Performed in the literature, multi-criteria building simulation based on short sequence has proved its efficiency in the domain of buildings and in reaching realistic performance. Short sequence could be found by different approaches that can be classified into three categories: Iterative, Heuristics and Grouping methods.

Parametric analysis after sampling with Latin Hypercube Sampling (LHS) had shown the ability of the iterative approach SCSPT in predicting annual performance of the energetic criteria of a solar combisystem. However, the approach did not work for all cases especially cases with large equipment.
And the results were found to be unstable when simulating same sequence on different models or same model on different sequences. In addition to that, some important criteria in such studies such as durability of the solar collectors by estimating moments of overheating could not be achieved. On the other hand, the issue of thermal inertia was also detected through the study, which would cause a problem in estimating the performance of models with high thermal inertia such as the summer comfort. So, the SCSPT method as it is, was found to be not stable and sufficient for such system models studies and further analysis is recommended.

This research work showed the advantage of the concept of model simulation based on short cycle sequences in the terms of the reduction of computational time expenses. Therefore, the domain of optimization might be a good field of interest where continuous varying of parameters takes place while searching for the optimal model. However, further improvements should be done in order to achieve better accuracy. For example, the criteria under interest should be used as selection criteria or performance criteria for better estimation by the reduction method. Therefore, the methods developed should cover a wider range of attributes than the ones covered by SCSPT. In addition to that, a better method of extrapolation is recommended for a fair representation of the temporal events and therefore a better prediction of the monthly and annual performances. From this point, the iterative approach and the clustering algorithm are chosen for the development of a new hybrid approach estimated to be capable of reproducing annual performance of building model. While the iterative approach will be responsible of dividing continually the annual data into portions of noticeable variations, the clustering algorithm will search for the center in each portion. Extrapolation will later be done based on the weight of each group so each group will be fairly represented. On the other hand, it is expected that thermal inertia might pose a problem in good annual estimation especially in the case of buildings' envelopes. Therefore, this phenomenon will be under examination through the coming studies.

\section{References}

Albaric M., Nowag, J. \& Papillon P., (2008). Thermal performance evaluation of solar combisystems using a global approach, EUROSUN2008, oct. 2008.

Balachandra P. \& Chandru V., (1999). Modelling electricity demand with representative load curves, Energy, vol. 24, no 3, p. 219-230.

Belderbos A. \& Delarue E., (2015). Accounting for flexibility in power system planning with 
renewables, Int. J. Electr. Power Energy Syst., vol. 71, p. 33-41.

Cheze D., Papillon P., Leconte A., Haller M.Y., Haberl R., Persson T., Bales C., Haberl R. and (2014). Towards an harmonized whole system test method for combined renewable heating systems for houses. EUROSUN2014, sep 2014.

Fazlollahi S., Bungener S.L., Mandel P., Becker G. \& Maréchal F., (2014). Multi-objectives, multi period optimization of district energy systems:I. Selection of typical operating periods, Comput. Chem. Eng., vol. 65, p. 54-66.

Fripp M., (2012). Switch: A Planning Tool for Power Systems with Large Shares of Intermittent Renewable Energy, Environ. Sci. Technol., vol. 46, no 11, p. 6371-6378.

Hart E.K. \& Jacobson M.Z., (2011). A Monte Carlo approach to generator portfolio planning and carbon emissions assessments of systems with large penetrations of variable renewables, Renew. Energy, vol. 36, no 8, p. 2278-2286.

Kotzur L., Markewitz P., Robinius M., et Stolten D., (2018). Impact of different time series aggregation methods on optimal energy system design, Renew. Energy, vol. 117, p. 474-487.

Menegon D., Soppelsa A. \& Fedrizzi R., (2017). Development of a new dynamic test procedure for the laboratory characterization of a whole heating and cooling system, Appl. Energy, vol. 205, p. 976-990.

Ortiga J., Bruno J.C. \& Coronas A., (2011).Selection of typical days for the characterisation of energy demand in cogeneration and trigeneration optimisation models for buildings, Energy Convers. Manag., vol. 52, no 4, p. 1934-1942.

Pérez-Lombard L., Ortiz J. \& Pout C., (2008). A review on buildings energy consumption information, Energy Build., vol. 40, no 3, p. 394-398.

Ribault C.(2017). Multicriteria optimization method for design assistance for urban densification process, $\mathrm{PhD}$ thesis study. 